\title{
MUÑOZ-BASOLS, JAVIER, DIR. 2017. PORTAL DE LA LINGÜIISTICA HISPÁNICA / HISPANIC LINGUISTICS. EN LÍNEA: HTTP://HISPANICLINGUISTICS.COM/
}

\author{
Josh PRADA \\ Texas Tech University \\ j.delarosa-prada@ttu.edu
}

\begin{abstract}
Vivimos en tiempos digitales, y la naturaleza global de la tecnología ha otorgado a nuestra sociedad una conectividad e inmediatez sin precedentes a la hora de manejar y acceder información. Esta realidad también está presente en el entorno académico. Ejemplos incluyen el auge en COMAs (i.e., cursos online masivos abiertos), un uso extendido de plataformas digitales en forma de laboratorios online, la inclusión de wikis y otras herramientas para colaboración en línea y las aplicaciones móviles para el aprendizaje de lenguas. Igualmente, distintos departamentos universitarios y laboratorios han desarrollado sitios web que sirven como puntos de diseminación digital para información importante en el campo, enfocándose en distintos intereses académicos y áreas geográficas. El Portal de Lingüística Hispánica - Hispanic Linguistics (Muñoz-Basols y Gironzetti, 2017) emerge como iniciativa a este movimiento hacia la digitalización, y se sitúa como trabajo pionero en las humanidades digitales, proveyendo una infinidad de recursos gratuitos cuidadosamente seleccionados y claramente ordenados en una plataforma navegable e intuitiva.

Con un foco delimitado sobre el campo de la lingüística hispánica y escrito en español, el proyecto (www.hispaniclinguistics.com) nace como elemento acompañante del volumen Introducción a la Lingüística Hispánica Actual: Teoría y Práctica (Muñoz-Basols, Moreno, Taboada y Lacorte, 2017) a través de la colaboración de Javier Muñoz-Basols (Universidad de Oxford, Reino Unido) y Elisa Gironzetti (Universidad de Maryland, EE.UU.) con el apoyo de una beca Hispanex. El portal está abierto a cualquier usuario: no es necesario registrarse y es gratuito. A pesar de hacer referencia al volumen de MuñozBasols et al., el portal puede ser utilizado de manera independiente, ya que contiene lecturas, actividades y bibliografías de una forma integrada y claramente planteada. La interfaz del sitio es simple y accesible, extendiéndose a través de ocho secciones temáticas, equivalentes a los capítulos del libro de Muñoz-Basols y colegas. Las secciones se muestran horizontalmente en la parte superior de la página. Organizadas de izquierda a derecha encontramos: 1 - Conceptos fundamentales; 2 - Fonética y fonología; 3 - Mor-
\end{abstract}

Para citar esta reseña / To cite this book review: Prada, Josh (2017). Reseña de Javier MuñozBasols, (dir.) (2017): Portal de la lingüistica hispánica / Hispanic Linguistics. En línea:http:/hispaniclinguistics.com/. ELUA, 31: 397-400. doi:10.14198/ELUA2017.31.25

Enlace / Link: http://dx.doi.org/10.14198/ELUA2017.31.25 
fología; 4 - Sintaxis; 5 - Semántica y pragmática; 6 - Historia de la lengua; 7 - Variación; 8 - Adquisición. A excepción de una, estas secciones están subdivididas en ocho apartados: (i) una presentación donde se delinea el alcance temático de cada sección; (ii) un apartado de "actividades del libro"; (iii) un listado de enlaces a "recursos prácticos" en línea; (iv) un apartado con propuestas para investigación; (v) seguido de una lista de grupos de investigación centrados en las áreas relevantes a la sección; (vi) un glosario de conceptos y términos clave; y (vii) una bibliografía temática.

Un aspecto interesante de este portal posiblemente está en las posibilidades que ofrece a la hora de interactuar con la interfaz. Su organización es clara y los menús son consistentes en las distintas páginas que conforman el portal. En los apartados de presentación de cada una de las secciones temáticas, los autores han subrayado los términos clave. Al posar el cursor del ratón sobre uno de estos términos, aparece una pequeña ventana, la cual contiene, por un lado, su traducción al inglés, y por otro lado una definición. Por ejemplo, en la sección temática número 5 del portal -semántica y pragmática-, encontramos subrayados términos como "cortesía lingüística", "deixis" y "actos de habla". En algunos casos, los autores también han incluido referencias a trabajos seminales conectados al término o noción en cuestión. Sin duda, esta función será utilísima tanto para estudiantes como para profesores, permitiendo una lectura más exacta y un acceso a los textos relacionados más informado, gracias al apoyo del glosario virtual automático. Aquellos usuarios que adquieran el volumen de Muñoz-Basols et al. (2017), podrán apoyarse en el portal durante la lectura, facilitando así la comprensión de términos y posibilitando una aproximación más rigurosa al texto. Los usuarios que no empleen el texto se beneficiarán igualmente de un glosario automático, exacto y riguroso.

El segundo apartado que el usuario encontrará en cada una de las ocho secciones es el de "Actividades del libro". Siendo este portal un acompañante del volumen de Muñoz-Basols y colegas, encontraremos que esta sección no detalla los enunciados de las actividades presentes en el libro. Por ejemplo, en el apartado 3 - Morfología, encontramos "Actividad 1. Conceptos fundamentales sobre morfología" (p. 112), seguido de tres recursos en línea (uno en inglés y dos en español). Estos recursos complementan y expanden las explicaciones ofrecidas en el volumen y sirven como materiales extra relevantes a las actividades que este presenta. Por lo tanto, aquellos usuarios que no tengan acceso al volumen de Muñoz-Basols y colegas, deberán formular sus propias preguntas, o bien emplear los recursos ofrecidos como herramientas de trabajo independientes. De cualquier modo, cabe destacar que los autores ofrecen información exacta sobre los aspectos temáticos cubiertos por cada actividad, (por ejemplo "Actividades 4 y 5. Los afijos flexivos y derivativos (p. 113))", permitiendo al lector comprender la relevancia de los enlaces que acompañan a las preguntas. Existen cinco tipos de enlaces, cada uno marcado por un icono específico: páginas web, archivos en formato PDF, herramientas online (e.g., buscadores, programas de análisis sintáctico y morfológico), capítulos de manuales en versión web, bases de datos tipo corpus y audios. Si bien el apartado de actividades puede parecer incompleto al no especificar los enunciados de las actividades en cuestión, esta sección contiene una serie de herramientas en línea activas, concretas y muy diversas, organizadas conceptualmente, y listas para acceder y compartir por estudiantes y profesores. Los autores han incluido también hipervínculos desde los cuales el usuario puede bajar programas gratuitos, como Praat, y guías de instalación y uso en español. 
El tercer apartado presente en cada una de las secciones es el de "recursos prácticos". Este listado es innovador en cuanto a que reúne en una sola página numerosas herramientas web que permiten explorar conceptos, constructos y fenómenos lingüísticos de una forma interactiva, incluyendo desde vídeos acompañados por transcripciones (e.g., Spanish Learner Videos \& Transcriptions, en sección 8 - adquisición), y herramientas de análisis morfosintáctico computarizado (sección 4 - sintaxis), hasta un atlas lingüístico de entonación del español o un silabeador (sección 2 - fonología y fonética). Sin duda, contar con un sitio web donde encontrar una variedad de herramientas funcionales tan diversas como la del Portal de Lingüistica Hispánica hacen de este un recurso innovador y muy valioso para el campo. En "temas para investigar", el cuarto apartado presente en las secciones, los autores ofrecen una serie de ideas para proyectos de investigación, seguido de posibles direcciones para diseminar los resultados en el entorno de clase (e.g., una presentación oral, un reporte escrito). Cada una de estas ideas de investigación está seguida por un listado de recursos en línea que pueden ser de gran ayuda para el estudiante. El glosario automático también está presente en este apartado, con algunos términos subrayados, traducciones y definiciones disponibles por medio de un movimiento del cursor. Es importante resaltar que las ideas de temas para investigar son muy adecuadas para estudiantes avanzados especializados en lingüística a nivel de grado, al igual que para aquellos estudiantes de postgrado con menos dominio de ciertas áreas. Cabe recordar que el portal acompaña a un volumen de introducción al campo, y consistentemente, las ideas para investigación encajan con este nivel.

Es sin duda otro punto a favor de los autores, el haber contemplado la inclusión de enlaces a las páginas de grupos de investigación sobre los temas presentados en el portal desde una perspectiva internacional. Por ejemplo, en sección 7 - variación, los autores incluyen, en este orden, grupos de México, Colombia, Japón, España, Estados Unidos y Argentina. La utilidad de esta sección para los miembros del alumnado y profesorado interesados en seguir de cerca el trabajo de grupos especializados, o de encontrar posibles contactos para colaboraciones, estancias e intercambios, es excelente. El sexto apartado ofrece un listado en orden alfabético de los conceptos y términos clave centrales a cada sección. Como ocurre en otros apartados, el diseño de este listado permite al usuario acceder a la traducción (al inglés) y a la definición de cada término al posar el cursor sobre la entrada elegida. En algunos casos, los autores han incluido publicaciones influyentes en referencia al constructo. Por ejemplo, en "competencia comunicativa", los autores acompañan la definición con referencias a las dos publicaciones seminales de Hymes, en 1966 y en 1971. El último apartado presente en las secciones principales que forman este portal consiste en una bibliografía organizada de forma temática y cronológica. Las referencias incluyen volúmenes de diferentes envergaduras, manuales y artículos de investigación, todos axiomáticos para el campo de la lingüística hispánica. Esta sección también incluye hipervínculos, facilitando así el acceso a publicaciones y otros portales.

Además de los apartados descritos anteriormente, el portal cuenta con cuatro botones situados en la parte superior derecha. El primero contiene una descripción del portal. El segundo lleva al usuario a un glosario extenso de uso sencillo, con opción para búsqueda por palabra, por primera letra o por sección. Se incluye también una pequeña descripción del libro al que este portal acompaña, un hipervínculo a la introducción del volumen, y un segundo hipervínculo para su compra por medio de la página web de la publicadora, Routledge. 
El Portal de Lingüistica Hispánica representa un trabajo claramente cuidadoso, una organización excelente y una amplitud destacable, abarcando los temas centrales de la lingüística hispánica. Si bien este portal guarda una relación cercana en cuanto a estructura y contenido con el libro de Muñoz-Basols y colegas, este portal puede ser empleado de manera independiente, acompañando otros manuales o como guía temática para desarrollar cursos similares. Su utilidad estriba en las posibilidades que ofrece como fuente de recursos: la cantidad de elementos en línea gratuitos que los autores han integrado en este sitio hace de él un juego de herramientas conceptuales y temáticas generoso y excepcionalmente útil. Recomiendo este portal para profesores en áreas de trabajo tradicionalmente cercanas a la lingüística, como la filología y la traducción e interpretación, al igual que para aquellos cuyos intereses mantienen intersecciones más puntuales con el campo, como la psicología, la sociología o la enseñanza. Todos encontrarán en este portal pautas temáticas sólidas, listas de lecturas cuidadas y organizadas, e ideas para implementar en clase (tanto a la hora de enfocar las actividades como en proyectos más amplios). Para el alumnado universitario interesado en explorar y desarrollar capacidades fundamentales en el campo, esta es sin duda una herramienta extraordinaria. Por un lado, la plataforma puede proporcionar a aquellos estudiantes más determinados un plan de acción estructurado para perseguir de forma autodidacta sus objetivos educativos en el área de la lingüística. Por otro lado, quienes requieran un apoyo extra para enfrentarse a sus clases en esta área, encontrarán en el portal un aliado valiosísimo.

En resumen, el Portal de Lingüística Hispánica (www.hispaniclinguistics.com) ofrece una panorámica clara sobre las áreas clave del campo, proveyendo información amplia y profunda por medio de publicaciones, herramientas web, audios y vídeos sobre una plataforma navegable, de manera gratuita. El trabajo de Muñoz-Basols y Gironzetti es cuidadoso, exacto, demostrando no solo un dominio extenso y detallado del campo de la lingüística hispánica, sino una familiaridad con los recursos disponibles en las diferentes disciplinas que lo conforman, y una voluntad de facilitar la comunicación entre profesores y alumnado en esta área de trabajo. Sin lugar a duda, una contribución inesperada que será muy bien recibida. 